\title{
Resolving Extragalactic Star Clusters with HST/ACS
}

\author{
Søren S. Larsen \\ Astronomical Institute, Utrecht University, Princetonplein 5, NL-3584 CC, \\ Utrecht, The Netherlands, larsen@astro.uu.nl
}

\begin{abstract}
Summary. With HST, colour-magnitude diagrams (CMDs) can be obtained for young star clusters well beyond the Local Group. Such data can help constrain cluster ages and metallicities, and also provide a reference against which intermediateand high mass stellar models can be compared. Here, CMDs are presented for two massive $\left(>10^{5} M_{\odot}\right)$ clusters and compared with Padua and Geneva isochrones. The problem of the ratio of blue to red supergiants is also addressed.
\end{abstract}

Star clusters remain the best approximation provided by Nature to "simple stellar populations" and have a long history as important test labs for models of stellar evolution. The rich old globular clusters (GCs) in the Milky Way have played a vital role for testing and calibrating models for low-mass stars 11. However, observational tests of intermediate- and high mass stars remain more scarce. The problem is largely one of statistics: for any realistic stellar initial mass function (IMF), massive stars constitute only a small fraction of the total number of stars in a cluster, and have the shortest life times. Where evolved stars are concerned, this difficulty becomes especially acute: a $10^{5} M_{\odot}$ cluster with an age of $10^{7}$ years and a Kroupa IMF [5] is only expected to contain about 25 red supergiants, while a 10 Gyr old GC of the same mass contains $\sim 1000$ post-main sequence stars. Clearly, evolved massive stars are rare in typical open clusters, and even within the entire Local Group the number of potential targets is limited if one wishes to obtain useful constraints on quantities such as the ratio of blue to red supergiants.

Fortunately, several galaxies within distances of a few Mpc contain significant populations of young "massive" clusters (YMCs) with masses in excess of $10^{5} \mathrm{M}_{\odot}$ 7]. In such clusters, the late stages of stellar evolution start to be reasonably well sampled even for young $\left(\sim 10^{7}-10^{8}\right.$ years $)$ ages. With HST, colour-magnitude diagrams (CMDs) are within reach for some of these clusters, although care has to be taken in order to deal with the severe crowding. In the following, early results for two illustrative cases are presented.

\section{Case 1: A 35-50 Myr old cluster in NGC 1313}

The Magellanic-type galaxy NGC 1313 ( $D \sim 4.1 \mathrm{Mpc}$ ) is known from groundbased imaging to host several YMCs [7. The western part of the galaxy 
appears to have experienced a recent burst of star formation during which also a particularly massive star cluster was formed (\#379 in the list of [7). The cluster has an age of $\sim 50$ Myrs (see below), consistent with the peak of field star formation activity in the region 8. The integrated magnitude $M_{V}=-10.9$ corresponds to a mass of $175000 M_{\odot}$ for a Chabrier IMF [2, 1].

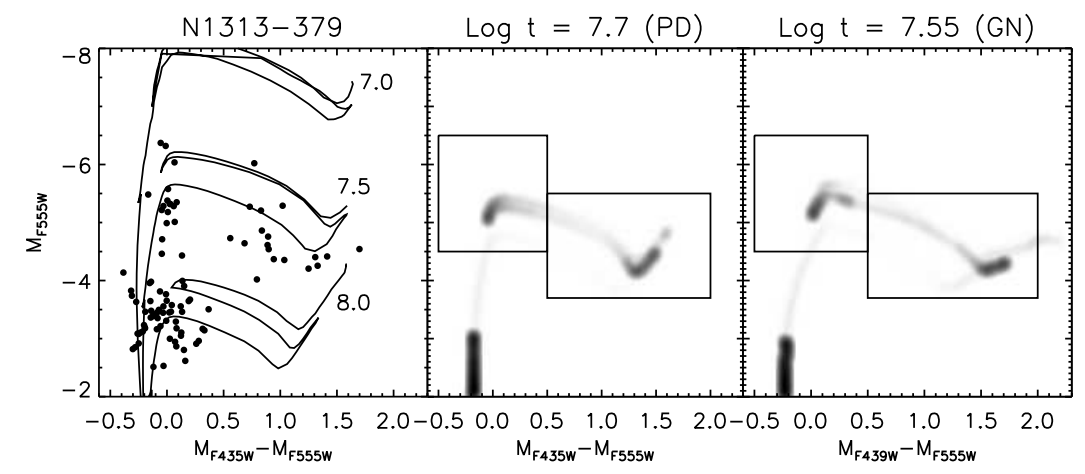

Fig. 1. Left: observed colour-magnitude diagram for a massive star cluster in NGC 1313. Centre and right: Hess diagrams showing the density of stars predicted by isochrones from the Padua (centre) and Geneva groups (right). Boxes indicate regions where red and blue supergiants are counted, as explained in the text.

The cluster is well resolved on our ACS/WFC images (Progr. ID 9774; P.I. S. S. Larsen), and the CMD is shown in Fig. 1(left). Several distinct features are seen: The main sequence turn-off (MSTO) is at $m_{\mathrm{F} 435}-m_{\mathrm{F} 555 \mathrm{~W}} \approx 0$ and $M_{V} \approx-3.5$. At brighter absolute magnitudes $\left(M_{V} \sim-5.5\right)$ are the blue core He burning stars ("blue supergiants", BSGs). The red core He burning stars ("red supergiants", RSGs) are seen at $m_{\mathrm{F} 435}-m_{\mathrm{F} 555 \mathrm{~W}}>0.5$ and $M_{V} \sim-4.5$. Also shown are Padua isochrones [4] for $Z=0.008$ and $\log t=7.0,7.5$ and 8.0. The cluster appears slightly older than $\log t=7.5$.

Isochrones do not fully illustrate the distribution of stars in the CMD. In particular, the gap between the MSTO and the BSGs is only revealed when the density of stars in the CMD is shown. The centre panel shows the synthetic Hess diagram for a $\log t=7.7$ Padua isochrone, while the righthand panel shows a $\log t=7.55$ Geneva isochrone. These ages correspond to MSTO masses of $7.4 M_{\odot}$ and $8.1 M_{\odot}$, respectively. Both sets of models reproduce the overall distribution of stars in the CMD fairly well, albeit for somewhat different ages. The RSGs may be slightly too red (cool) in both cases. The apparent difference in the model colours of the RSGs is mostly due to the fact that WFPC2 F439W-F555W colours are shown for the Geneva isochrones, while the proper ACS/WFC F435W-F555W colours are used for the Padua isochrones. In reality, the difference in $T_{\text {eff }}$ between the Padua and Geneva RSGs is small, with the Geneva RSGs being some $150 \mathrm{~K}$ cooler. 
One long-standing problem concerns the relative fraction of RSGs and BSGs 6, 3]. Models tend to predict a decreasing BSG/RSG ratio with increasing metallicity, while observations show the opposite. Fortunately, a comparison with the data is possible without an exact calibration between fundamental stellar properties $\left(L, T_{\text {eff }}\right)$ and colours. The boxes drawn in Fig. 1 indicate regions of the CMD where we count RSGs and BSGs. In the observed CMD we find 16 BSGs and 17 RSGs, corresponding to a ratio of $\mathrm{BSG} / \mathrm{RSG}=0.94 \pm 0.33$. Within the error, this is consistent with the model predictions: $\mathrm{BSG} / \mathrm{RSG}=0.73$ and 0.74 for the Padua and Geneva models.

To summarize, the agreement between models and observations seems rather satisfactory for this cluster. This is true both for the colours and luminosities of various types of stars and the BSG/RSG ratio. Since the stars in this cluster only barely qualify for the label "massive", this was perhaps to be expected. It should be noted, however, that the CMD-based age could be either 50 Myr or $35 \mathrm{Myr}$, depending on the choice of models. An important corollary is that absolute ages derived from integrated colours (or spectra) are likely to be uncertain by at least the same amount.

\section{Case 2: Cluster NGC 1569-B}

The dwarf irregular galaxy NGC 1569 is well known for hosting two very compact, massive young clusters. Although it is about a factor of two closer than NGC 1313, observations of the clusters in NGC 1569 are challenging due to both their more compact structure, the general degree of crowding, and the significant Galactic foreground reddening $\left(b=11^{\circ}\right)$. Nevertheless, both clusters A and B are resolved into individual stars in ACS/HRC data (Progr. ID. 9300; P.I. H. Ford); here we concentrate on cluster B for which we also have Keck/NIRSPEC near-infrared spectroscopy [9]. The reddeningcorrected absolute $V$ magnitude is $M_{V}=-12.2$ [10, corresponding to a mass of about $280000 M_{\odot}$ for an age of 15 Myrs.

The CMD for NGC 1569-B is shown in Fig. 2. The RSGs can again be clearly discerned, but the identification of BSGs is less obvious and the MSTO is too faint to be detected. The best fitting Padua models have an age of $\log t=7.25$ while the best fitting Geneva models are slightly younger; $\log t=$ 7.15. The corresponding MSTO masses are $13 M_{\odot}$ and $14 M_{\odot}$, respectively. We have again assumed $Z=0.008$ models, which give the best fits, although this may not exactly match the actual metallicity of NGC 1569. However, neither set of models provides a very good match to the observations for any metallicity. Even for fairly generous limits in the selection of BSGs, the observed BSG/RSG ratio of 28/60 = 0.47 \pm 0.11 is well below the model predictions (BSG/RSG $=1.28$ and 0.87 for the Padua and Geneva models). The CMD also shows a small number of very bright $\left(M_{V}<-8\right)$, blue stars which are not predicted by any model for this age. These are redder than the fainter blue stars, and thus unlikely to be simple blends. One possibility is 


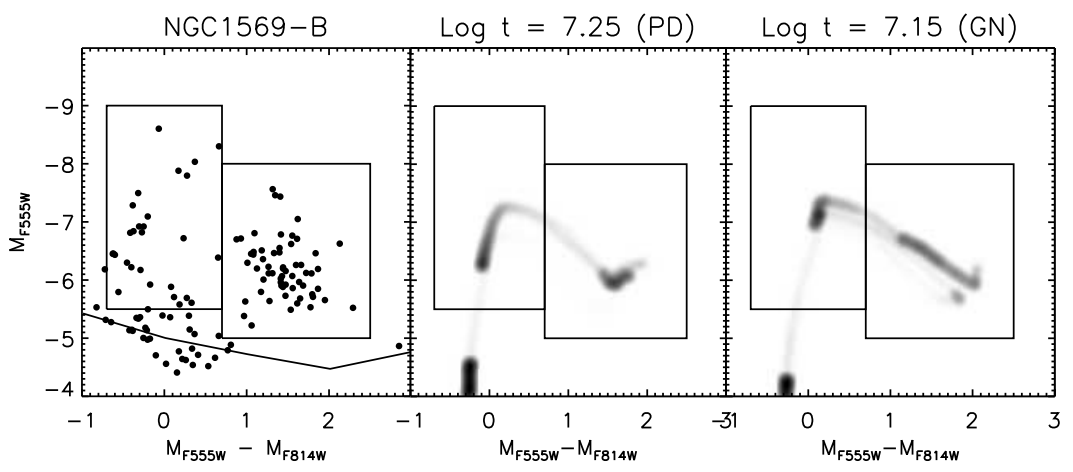

Fig. 2. As Fig. 1 but for NGC 1569-B. The $75 \%$ completeness limit is indicated in the left-hand panel.

that these stars have somewhat younger ages than the majority of stars in cluster B.

\section{Concluding remarks}

The two cases discussed here demonstrate that useful information can be extracted from the CMDs of star clusters well beyond the Local Group. Young, massive $\left(>10^{5} M_{\odot}\right)$ clusters offer significant samples of stars with similar age and chemical composition, offering a potentially powerful way to test models for massive stars. Most current alternatives either suffer from small number statistics (e.g. open clusters in the Milky Way) or from difficulties disentangling the contributions from stars of different ages, hence masses (e.g. resolved stellar populations in Local Group galaxies).

\section{References}

1. Bruzual, G., Charlot, S., 2003, MNRAS, 344, 1000

2. Chabrier, G., 2003, PASP, 115, 763

3. Eggenberger, P., et al., 2002, A\&A, 386, 576

4. Girardi, L., et al. 2002, A\&A, 391, 195

5. Kroupa, P. 2002, Science, 295, 82

6. Langer, N., Maeder, A. 1995, A\&A, 295, 685

7. Larsen, S. S., Richtler, T. 1999, A\&A, 345, 59

8. Larsen, S. S., et al. 2007, in IAU Symp. 241, ed. A. Vazdekis and R. Peletier (astro-ph/0701697)

9. Larsen, S. S., Origlia, L., et al. 2007, MNRAS, in prep.

10. Origlia, L., et al. 2001, AJ, 122, 815

11. Renzini, A., Fusi Pecci, F. 1988, ARA\&A, 26, 199 